\title{
PR interval prolongation in coronary patients or risk equivalent: excess risk of ischemic stroke and vascular pathophysiological insights
}

Yap-Hang Chan ${ }^{1}$ (D, Jo Jo Hai ${ }^{1}$, Kui-Kai Lau², Sheung-Wai Li ${ }^{3}$, Chu-Pak Lau', Chung-Wah Siư ${ }^{1,4}$, Kai-Hang Yiu ${ }^{1}$ and Hung-Fat Tse ${ }^{1,4^{*}}$

\begin{abstract}
Background: Whether PR prolongation independently predicts new-onset ischemic events of myocardial infarction and stroke was unclear. Underlying pathophysiological mechanisms of PR prolongation leading to adverse cardiovascular events were poorly understood. We investigated the role of PR prolongation in pathophysiologically-related adverse cardiovascular events and underlying mechanisms.

Methods: We prospectively investigated 597 high-risk cardiovascular outpatients (mean age $66 \pm 11$ yrs.; male 67\%; coronary disease $55 \%$, stroke $22 \%$, diabetes 52\%) for new-onset ischemic stroke, myocardial infarction (MI), congestive heart failure (CHF), and cardiovascular death. Vascular phenotype was determined by carotid intima-media thickness (IMT).

Results: PR prolongation >200 ms was present in 79 patients (13\%) at baseline. PR prolongation $>200$ ms was associated with significantly higher mean carotid IMT $(1.05 \pm 0.37 \mathrm{~mm}$ vs $0.94 \pm 0.28 \mathrm{~mm}, P=0.010)$. After mean study period of $63 \pm 11$ months, increased PR interval significantly predicted new-onset ischemic stroke $(P=0.006)$, CHF $(P=0.040)$, cardiovascular death $(P<0.001)$, and combined cardiovascular endpoints $(P<0.001)$ at cut-off $>200 \mathrm{~ms}$. Using multivariable Cox regression, PR prolongation $>200 \mathrm{~ms}$ independently predicted new-onset ischemic stroke (HR 8.6, 95\% Cl: 1.9-37.8, $P=0.005$ ), cardiovascular death (HR 14.1, 95\% Cl: 3.8-51.4, $P<0.001$ ) and combined cardiovascular endpoints ( $\mathrm{HR} 2.4,95 \% \mathrm{Cl}: 1.30-4.43, P=0.005)$. PR interval predicts new-onset $\mathrm{Ml}$ at the exploratory cut-off $>162 \mathrm{~ms}$ (Cstatistic $0.70, P=0.001 ; \mathrm{HR}: 8.0,95 \%$ Cl: $1.65-38.85, P=0.010)$.

Conclusions: PR prolongation strongly predicts new-onset ischemic stroke, $\mathrm{MI}$, cardiovascular death, and combined cardiovascular endpoint including CHF in coronary patients or risk equivalent. Adverse vascular function may implicate an intermediate pathophysiological phenotype or mediating mechanism.
\end{abstract}

Keywords: PR interval prolongation, Cardiovascular death, Myocardial infarction, Ischemic stroke, Carotid intima-media thickness, Vascular function, Pathophysiological mechanism

\footnotetext{
* Correspondence: hftse@hkucc.hku.hk

'Division of Cardiology, Department of Medicine, Queen Mary Hospital,

University of Hong Kong, Rm 1928, Block K, Hong Kong, China

${ }^{4}$ Research Center of Heart, Brain, Hormone and Healthy Ageing, University of

Hong Kong, Hong Kong, China

Full list of author information is available at the end of the article
} 


\section{Background}

First-degree heart block, referred to as prolongation of $\mathrm{PR}$ interval beyond $>200 \mathrm{~ms}$ on the electrocardiogram (ECG), has long been considered a benign functional variation [1]. Nevertheless, recent major studies including the ARIC [2] and Framingham Heart Study [3], re-ignited controversies over the pathological nature of PR prolongation by consistently demonstrating that PR prolongation was linked to risk of atrial fibrillation in the population. In the Framingham study, PR prolongation was further associated with increased risk of pacemaker implantation and all-cause death [3]. Nevertheless, the roles of PR prolongation in atherosclerotic cardiovascular $(\mathrm{CV})$ disease, as well as outcome prediction in $\mathrm{CV}$ patients under clinical care, remained largely unknown. The Heart and Soul Study showed that PR prolongation predicted heart failure hospitalization and CV mortality in patients with stable coronary artery disease (CAD) [4]. We further showed that PR prolongation when incorporated into the CHADS2 and CHA2DS2-VASc scores, augments their predictive power for new-onset CV events [5]. Nevertheless, the individual role of PR prolongation, a precursor to atrial fibrillation, for new-onset ischemic events of stroke and myocardial infarction was not clearly established. Importantly, underlying pathophysiological mechanistic basis of how PR prolongation may lead to adverse $\mathrm{CV}$ outcomes remained also poorly understood.

In a prior study of 88 healthy subjects, PR prolongation was found independently correlated with endothelial dysfunction and increased pulse-wave velocity even in the absence of clinically manifest atherosclerotic disease [6], suggesting that adverse vascular function could be an intermediate pathological phenotype in subjects with PR prolongation progressing towards clinical events. As endothelial dysfunction indicates widespread functional adversity of the CV system as a collective, it will be of interest to dissect the role of PR prolongation in closely related $\mathrm{CV}$ pathological entities commonly characterized by abnormal vascular function, including myocardial infarction (MI) [7], ischemic stroke [8], and congestive heart failure (CHF) [9].

Therefore, we investigated in this prospective cohort study the role of $\mathrm{PR}$ prolongation as an independent predictor in a continuum of pathophysiologically-linked clinical events including ischemic stroke, MI, CHF, and $\mathrm{CV}$ death in high-risk CV patients, and the relationships between PR prolongation and indicator of abnormal vascular function.

\section{Methods}

\section{Study population, design and clinical assessments}

We studied 597 consecutive patients with CAD or risk equivalent recruited from outpatient medical specialty clinics, as prior described [5]. Among them, 328 patients had history of prior CAD (55\%), 131 had ischemic stroke (22\%), and 310 had diabetes mellitus (52\%). All patients completed written informed consent. The study was adherent to the Declaration of Helsinki and was approved by the Ethics Committee, Hospital Authority (Hong Kong West)/University of Hong Kong.

During the study period Dec 2007 to May 2012, occurrence of $\mathrm{CV}$ death, new-onset ischemic stroke,

$\mathrm{MI}$ and $\mathrm{CHF}$ were retrieved from the central computerized clinical records archive of all public hospitals. Primary endpoints of the study were carotid IMT and clinical events of $\mathrm{CV}$ death, new-onset MI and ischemic stroke. Secondary Endpoints were new-onset CHF and combined CV endpoints, which included occurrences of any primary endpoints or CHF. Baseline demographic, clinical and laboratory assessments, clinical event definitions, and the inclusion/exclusion criteria were prior detailed elsewhere [5].

\section{Carotid Intima-Media Thickness (IMT)}

As previously described in details [10], carotid IMT was assessed as the distance between lumen-intima and mediaadventitia borders of the carotid arterial wall using electronic calipers under high-resolution ultrasound. Three measurements were taken at multiple sites on each side of the carotid arteries with resultant averaged estimate incorporating all measurements. Intraobserver variability analyses revealed satisfactory correlation $(R=0.97, P<0.001)$.

\section{PR prolongation}

Each participant had 12-lead ECG assessment after rested in the supine position for $5 \mathrm{~min}$. PR interval prolongation was defined as $>200 \mathrm{~ms}$ [1]. An exploratory cut-off of PR interval > $162 \mathrm{~ms}$ was further tested based on the median cut-off correlated with endothelial dysfunction in our earlier exploratory study of 88 healthy individuals [6]. Widened QRS duration was determined as $>120 \mathrm{~ms}$ [11].

\section{Statistical analysis}

Associations between PR interval and carotid IMT were examined by univariable and multivariable linear regression. Kaplan-Meier analysis was used to assess differences in survival as stratified by baseline PR interval status. A multivariable Cox proportional regression model was used to derive Hazard Ratios (HR) for incident CV events such that potential confounder variables with $p$-value $\leq 0.20$ (based on univariable analysis) were entered. Findings were compared with aother Fully-Adjusted Model to verify robustness of study findings, such that variables a priori determined to be relevant were included (Additional file 1). The Vascular Function Model further dissects effect of carotid IMT adjustment in the estimates of PR interval as 
an independent predictor for CV events. SPSS Statistics (Version 20) was used for the above analyses.

\section{Results}

\section{Baseline clinical characteristics}

As shown in Table 1, PR prolongation $>200 \mathrm{~ms}$ was present in $13 \%$ of patients (79/597). Patients with PR prolongation had higher mean age $(P<0.001)$ and greater male predominance $(P<0.001)$. There were no

Table 1 Baseline characteristics of Participants by PR Interval Status $(n=597)^{\text {a }}$

\begin{tabular}{|c|c|c|c|}
\hline & $\begin{array}{l}\text { PR Interval } \\
\leq 200 \mathrm{~ms} \\
(n=518)\end{array}$ & $\begin{array}{l}\text { PR Interval } \\
>200 \mathrm{~ms} \\
(n=79)\end{array}$ & $p$-value \\
\hline Male [n (\%)] & $334(65 \%)$ & $67(85 \%)$ & $<0.001^{*}$ \\
\hline Age (years) & $65.0 \pm 11.1$ & $70.5 \pm 9.6$ & $<0.001^{*}$ \\
\hline Body mass index $\left(\mathrm{kgm}^{-2}\right)$ & $25.2 \pm 3.5$ & $25.6 \pm 3.2$ & 0.34 \\
\hline Coronary Artery Disease [n (\%)] & $276(53 \%)$ & $52(66 \%)$ & $0.037^{*}$ \\
\hline Prior stroke [n (\%)] & $105(21 \%)$ & $26(33 \%)$ & $0.017^{*}$ \\
\hline Diabetes mellitus [n (\%)] & $274(53 \%)$ & $36(46 \%)$ & 0.23 \\
\hline Hypertension [n (\%)] & $330(65 \%)$ & $57(72 \%)$ & 0.20 \\
\hline Hyperlipidemia [n (\%)] & $320(64 \%)$ & $47(60 \%)$ & 0.48 \\
\hline Current/Past smoker [n (\%)] & $211(42 \%)$ & $43(54 \%)$ & 0.042 \\
\hline Regular Physical activity [n (\%)] & $185(37 \%)$ & $25(12 \%)$ & 0.40 \\
\hline Resting pulse rate (/min) & $60.3 \pm 21.2$ & $63.7 \pm 15.4$ & 0.18 \\
\hline Systolic blood pressure $(\mathrm{mmHg})$ & $141.1 \pm 20.5$ & $140.2 \pm 18.6$ & 0.71 \\
\hline Diastolic blood pressure $(\mathrm{mmHg})$ & $79.0 \pm 9.6$ & $78.7 \pm 9.3$ & 0.83 \\
\hline LDL-cholesterol (mmol/L) & $2.7 \pm 0.7$ & $2.4 \pm 0.70$ & $0.001 *$ \\
\hline HDL-cholesterol (mmol/L) & $1.3 \pm 0.3$ & $1.2 \pm 0.4$ & 0.14 \\
\hline Tiglycerides (mmol/L) & $1.5 \pm 1.0$ & $1.4 \pm 0.7$ & 0.37 \\
\hline Fasting glucose (mmol/L) & $6.3 \pm 2.1$ & $6.1 \pm 1.9$ & 0.32 \\
\hline HbA1c (\%) & $7.0 \pm 1.5$ & $6.8 \pm 1.2$ & 0.22 \\
\hline hs-CRP (mg/L) & $3.0 \pm 8.9$ & $2.7 \pm 5.7$ & 0.80 \\
\hline Serum creatinine $(\mu \mathrm{mol} / \mathrm{L})$ & $86.9 \pm 29.2$ & $106.9 \pm 59.6$ & $0.005^{*}$ \\
\hline \multicolumn{4}{|l|}{ Medications: } \\
\hline ACEI/ARB [n (\%)] & $268(54 \%)$ & $55(70 \%)$ & $0.008^{*}$ \\
\hline Beta-blockers [n (\%)] & $241(49 \%)$ & $43(54 \%)$ & 0.39 \\
\hline Calcium channel blockers [n (\%)] & $140(28 \%)$ & $22(28 \%)$ & 0.97 \\
\hline Aspirin [n (\%)] & $323(65 \%)$ & $62(79 \%)$ & $0.017^{*}$ \\
\hline Statin $[n(\%)]$ & $285(57 \%)$ & $49(62 \%)$ & 0.41 \\
\hline PR interval (ms) & $164.7 \pm 19.0$ & $222.6 \pm 22.0$ & $<0.001^{*}$ \\
\hline QRS duration (ms) & $94.3 \pm 27.1$ & $99.0 \pm 17.8$ & 0.14 \\
\hline Mean Carotid IMT (mm) & $0.94 \pm 0.28$ & $1.05 \pm 0.37$ & $0.010^{*}$ \\
\hline
\end{tabular}

$H D L$ high-density lipoprotein, $L D L$ low-density lipoprotein, HbA1c glycosylated haemoglobin A1c, hs-CRP high-sensitivity C-reactive protein, ACEI angiotensinconverting enzyme inhibitors, $A R B$ angiotensin receptor blockers, IMT intima-media thickness

* $P$-value $<0.05$

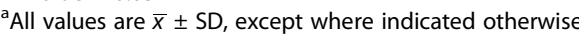

significant differences in history of diabetes mellitus, hyperlipidemia, smoking, physical activity, resting pulse rate, systolic/diastolic blood pressure, fasting glucose, HbA1c, and hsCRP (all $P>0.05$ ). Lower LDL-cholesterol was noted in patients with PR prolongation $(P<0.001)$ with no differences in triglycerides and HDL-cholesterol $(P>0.05)$, which could be partially due to higher prevalence of statin use among patients with PR prolongation, albeit that was not statistically significant $(P=0.41)$. PR prolongation was associated with higher serum creatinine indicating impaired renal function $(106.9 \pm 59.6$ versus $86.9 \pm 29.2 \mathrm{mmol} / \mathrm{L}, P=0.005)$, and a higher proportion of patients with $\mathrm{PR}$ prolongation had prior CAD $(P=0.037)$ or stroke $(P=0.017)$, with expectedly more prevalent use of aspirin $(P=0.017)$ and ACEI/ARB $(P=0.008)$. No between-groups difference of PR interval were found regarding the use of nodal-blocking agents including beta-blockers (use versus no use: $174.6 \pm 27.1 \mathrm{~ms}$ versus $171.2 \pm 28.5 \mathrm{~ms}, P=0.15$; linear regression $(\mathrm{B}=+3.3$ [95\% CI: $-1.2-7.9$ ], $P=0.39$ ); and CCB (use versus no use: $173.7 \pm 26.5 \mathrm{~ms}$ versus $172.2 \pm 28.3 \mathrm{~ms}$, $P=0.54$; linear regression $(\mathrm{B}=+1.6$ [95\% CI: $-3.5-6.6$, $P=0.54)$. QRS duration was similar between groups $(P=0.14)$.

\section{PR prolongation and adverse vascular function}

In this group of high-risk patients with CAD or risk equivalent, increased PR interval was positively associated with increased carotid IMT (Pearson $R=0.13$, $P=0.002$ ). Patients with PR prolongation had significantly higher mean carotid IMT $(1.05 \pm 0.37$ versus $0.94 \pm 0.28 \mathrm{~mm}, P=0.010$, Fig. 1). Adjusted for potential confounding variables including age, gender, prior stroke, smoking, body-mass index, resting pulse rate, systolic blood pressure, hs-CRP, fasting glucose, creatinine, and use of ACEI/ARB, CCB, beta-blockers, aspirin, and widened QRS interval, PR prolongation $>200 \mathrm{~ms}$ remained independently associated with higher carotid IMT by $+0.073 \mathrm{~mm}(95 \%$ CI: $0.003-0.143, P=0.041$, Additional file 1: Table S1). Repeated analysis by the Fully-Adjusted model produced similar results $(\mathrm{B}=+0.074$ [95\% CI: 0.002-0.147], $P=0.044$ ). Other positive predictors for carotid IMT included age, prior stroke and systolic blood pressure. Statin use was independently associated with lower carotid IMT $(P=0.041)$. Diastolic blood pressure was no longer a significant predictor after adjusting for pulse pressure in relation to systolic blood pressure $(P>0.05)$. QRS duration was not associated with carotid $\operatorname{IMT}(R=0.03, P=0.55)$.

\section{Prediction estimate of PR prolongation for new-onset ischemic stroke and CV events}

After mean study period of $63.4 \pm 10.6$ months, 21 (4\%) patients developed new-onset ischemic stroke; $26(4 \%)$ 


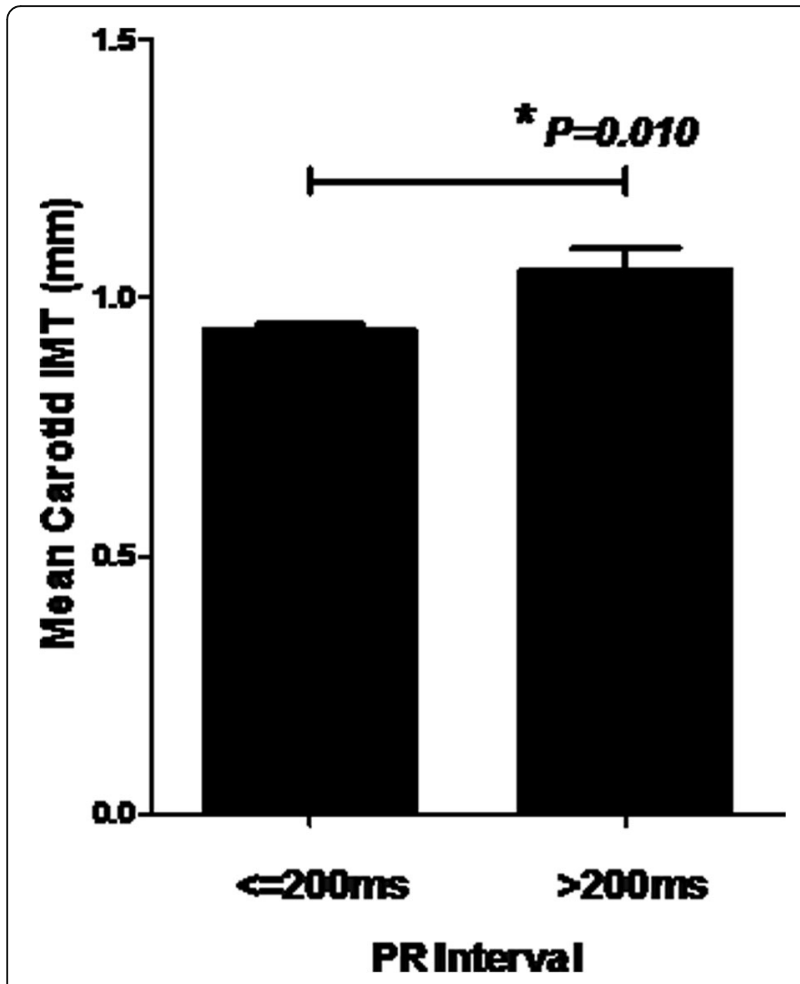

Fig. 1 PR Prolongation and Mean Carotid Intima-Media Thickness (IMT). Patients with PR prolongation $>200$ ms had significantly higher mean carotid IMT $(1.05 \pm 0.37$ versus $0.94 \pm 0.28 \mathrm{~mm}, P=0.010)$ patients developed new-onset MI; 37 (6\%) patients developed new-onset CHF; 27 (5\%) patients had CV death and 87 (19\%) patients developed any of these adverse CV events as a combined endpoint (Table $2 \mathrm{a}$ and $\mathrm{b}$ ). Patients with PR prolongation $>200 \mathrm{~ms}$ at baseline had significantly higher risk of new-onset ischemic stroke $(P=0.006)$, CHF $(P=0.039), \mathrm{CV}$ death $(P<0.001)$, and combined $\mathrm{CV}$ endpoint $(P<0.001)$. PR interval length was not associated with risk of new-onset MI at cut-off $>200 \mathrm{~ms}$ $(P>0.05)$ (primary cut-off) but a positive association was observed at the exploratory cut-off $>162 \mathrm{~ms}$ $(P=0.008)$. ROC Curve analysis revealed overall significant prediction of PR interval for new-onset MI (Cstatistic $0.70, P=0.001$ ).

\section{CV death}

Incidence rates for $\mathrm{CV}$ death in patients with $\mathrm{PR}$ interval $\leq 200 \mathrm{~ms}$ or $>200 \mathrm{~ms}$ were respectively 0.6 and 1.8 per 100 person-years. Univariable analysis revealed that age, resting pulse rate, systolic blood pressure, serum hs-CRP, creatinine, widened QRS > $120 \mathrm{~ms}$, and PR prolongation were all significant positive predictors for CV death (all $P<0.05$ ). Furthermore, KaplanMeier analysis showed that subjects with PR prolongation $>200 \mathrm{~ms}$ had significantly reduced survival (log rank: 14.4, $P<0.001$, Fig. 2a). Multivariable Cox regression analysis showed that adjusted for potential confounders, PR prolongation $>200 \mathrm{~ms}$ independently predicted substantially increased CV death (HR 6.53, 95\% CI: 2.39-17.84, $P<0.001$, Additional file 1: Table S2). Analyses by the Fully-Adjusted Model yielded similarly robust estimates (HR: 14.94, 95\% CI: 3.99-55.92, $P<0.001$ ). The

Table 2 New-Onset Cardiovascular (CV) Events Stratified by Baseline PR Interval and QRS Duration

\begin{tabular}{|c|c|c|c|c|c|c|}
\hline \multicolumn{7}{|l|}{ (a). PR interval } \\
\hline \multirow[t]{2}{*}{ New-Onset CV Events n (\%) } & \multirow[t]{2}{*}{ Total $(n=597)$} & \multirow[t]{2}{*}{ PR Interval < 200 ms $(n=518)$} & \multirow[t]{2}{*}{ PR Interval > 200 ms $(n=79)$} & \multirow[t]{2}{*}{$P$-value } & \multicolumn{2}{|c|}{ ROC C-Statistic } \\
\hline & & & & & Estimate & P-value \\
\hline Myocardial infarction & $26(4 \%)$ & $20(4 \%)$ & $6(8 \%)$ & 0.130 & 0.70 & $0.001^{*}$ \\
\hline Ischemic stroke & $21(4 \%)$ & $14(3 \%)$ & 7 (9\%) & $0.006^{*}$ & 0.50 & 0.99 \\
\hline Congestive heart failure & $37(6 \%)$ & $28(5 \%)$ & $9(11 \%)$ & $0.040^{*}$ & 0.60 & $0.042^{*}$ \\
\hline CV death & $27(5 \%)$ & $17(3 \%)$ & $10(13 \%)$ & $<0.001^{*}$ & 0.63 & $0.020^{*}$ \\
\hline Combined CV endpoints & 87 (19\%) & $65(13 \%)$ & $22(28 \%)$ & $<0.001^{*}$ & 0.61 & $0.001^{*}$ \\
\hline \multicolumn{7}{|l|}{ (b). QRS duration } \\
\hline \multirow[t]{2}{*}{ New-Onset CV Events n (\%) } & & QRS Duration $<120$ ms $(n=556)$ & QRS Duration >120 ms $(n=36)$ & $P$-value & \multicolumn{2}{|c|}{ ROC C-Statistic } \\
\hline & & & & & Estimate & $P$-value \\
\hline Myocardial infarction & - & $22(4 \%)$ & $3(8 \%)$ & 0.21 & 0.55 & 0.37 \\
\hline Ischemic stroke & - & $20(4 \%)$ & $1(3 \%)$ & 0.80 & 0.47 & 0.60 \\
\hline Congestive heart failure & - & $30(5 \%)$ & $7(19 \%)$ & $0.001^{*}$ & 0.59 & 0.08 \\
\hline CV death & - & $24(4 \%)$ & $3(8 \%)$ & 0.26 & 0.62 & $0.03^{*}$ \\
\hline Combined CV endpoints & - & $76(14 \%)$ & $10(28 \%)$ & $0.020^{*}$ & 0.56 & 0.08 \\
\hline
\end{tabular}



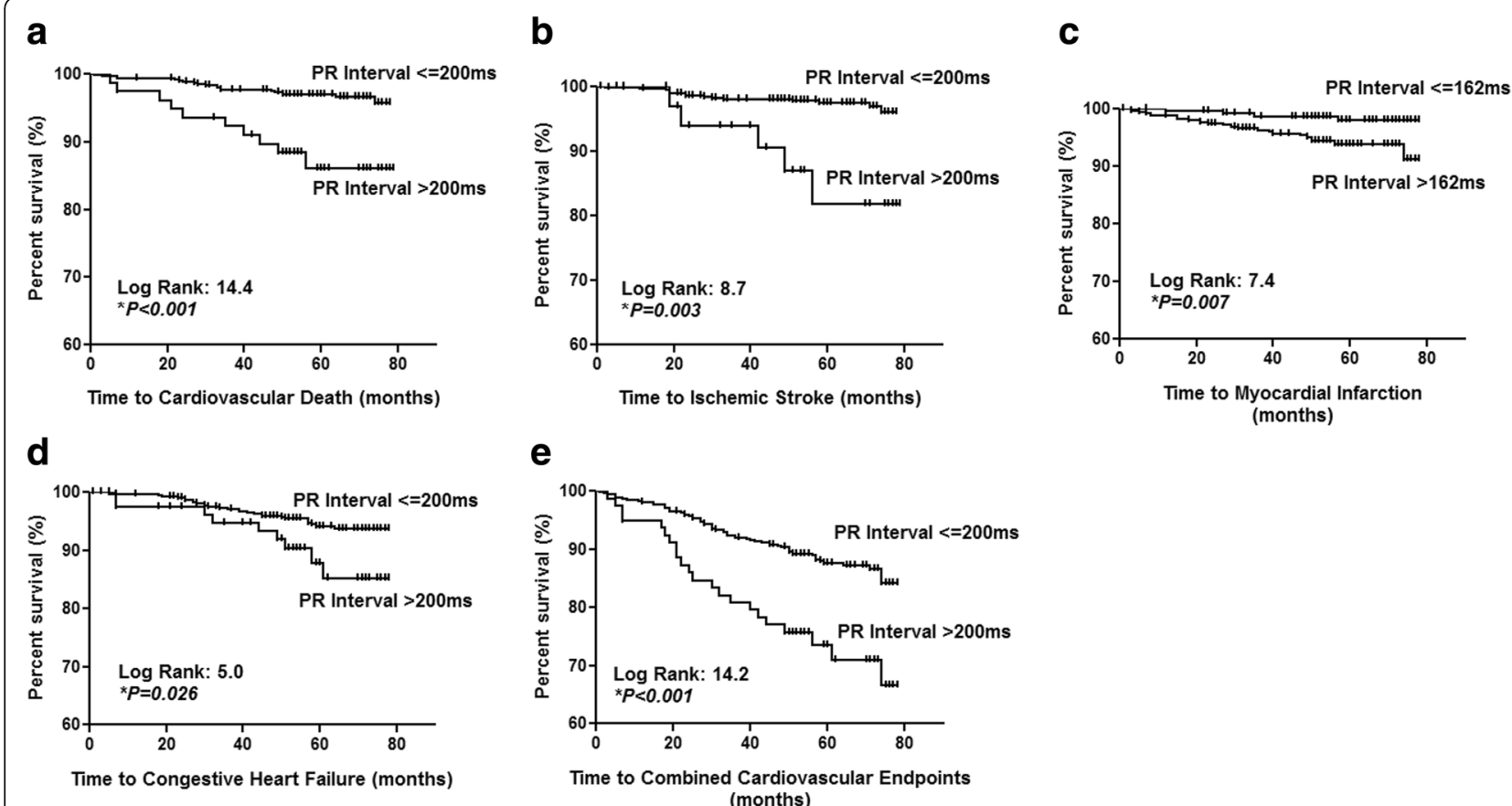

Fig. 2 Kaplan-Meier Survival Curves for New-Onset Cardiovascular Events by PR Prolongation. Patients with PR prolongation $>200$ ms had significantly reduced survival from a cardiovascular death (log rank: 14.4, $P<0.001$ ); b Ischemic Stroke (log rank: 8.7, $P=0.003$ ); d CHF (log rank: 5.0, $P=0.026)$ e Combined Cardiovascular Endpoints (log rank: 14.2, $P<0.001$ ). PR interval $>162$ ms was associated with reduced survival from c new-onset MI (log rank: $7.4, P=0.007)$

Vascular Function Model showed that adjusting for carotid IMT did not materially alter the independent prediction by PR prolongation (HR: 16.40, 3.99-67.46, $P<0.001$ ). Other independent positive predictors included HbAlc and hsCRP (both $P<0.05$ ). Higher BMI was associated with reduced $\mathrm{CV}$ death $(P<0.05)$. On the other hand, PR interval predicted total mortality with only marginal statistical significance (C-statistic 0.58, $P=0.042)$, and that it did not predict non- $\mathrm{CV}$ death $(\mathrm{C}$-statistic $0.53, P=0.63)$.

\section{Ischemic stroke}

Incidence rates for ischemic stroke in patients with $P R$ interval $\leq 200 \mathrm{~ms}$ or $>200 \mathrm{~ms}$ were respectively 0.5 and 1.9 per 100 person-years. Age, serum creatinine, carotid IMT, and PR prolongation $>200 \mathrm{~ms}$ were all significant predicting variables for new-onset ischemic stroke (all $P<0.05)$. Furthermore, Kaplan-Meier analysis showed that PR prolongation $>200 \mathrm{~ms}$ was significantly associated with reduced new-onset ischemic stroke-free survival (log rank: $8.7, P=0.003$, Fig. $2 b$ ). By multivariable Cox regression, PR prolongation $>200 \mathrm{~ms}$ independently predicted increased risk of new-onset ischemic stroke (HR 3.76, 95\% CI: 1.27-11.12, $P=0.016$, Additional file 1: Table S3). Analysis using the Fully-Adjusted Model gave similarly robust estimates (HR: 5.36, 95\% CI: 1.47-19.5, $P=0.011$ ). Adjusting for carotid IMT in the Vascular Function
Model, the independent predictive estimate by PR prolongation was only slightly attenuated (HR: $5.05,95 \%$ CI: 1.34-19.10, $P=0.017$ ).

\section{MI}

Incidence rates for $\mathrm{MI}$ in patients with $\mathrm{PR}$ interval $\leq 162 \mathrm{~ms}$ or $>162$ ms were 0.3 and 1.3 per 100 person-years respectively. Age, prior CAD, resting pulse rate, systolic blood pressure, serum creatinine, history of ACEI/ARB use, carotid IMT, and PR prolongation $>162 \mathrm{~ms}$ (exploratory cut-off) were all significant positive predictors for newonset MI (all $P<0.05$ ). However, the primary cut-off of PR interval $>200 \mathrm{~ms}$ was not associated with new-onset MI $(P=0.11)$. Kaplan-Meier analysis showed that patients with PR prolongation $>162 \mathrm{~ms}$ had significantly reduced new-onset MI-free survival (log rank: 7.4, $P=0.007$, Fig. 2c). Adjusting for potential confounders, PR prolongation $>162 \mathrm{~ms}$ independently predicted increased risk of new-onset MI (HR: 8.0, 95\% CI: 1.65-38.85, $P=0.010$, Additional file 1: Table S4). Repeated analysis using the Vascular Function Model did not materially alter the independent prediction of new-onset MI by PR prolongation $>162 \mathrm{~m}$ (HR: 8.05 , 95\% CI: 1.66-39.06, $P=0.010)$. Other independent positive predictors for MI included age and prior CAD. BMI was associated with reduced new-onset MI $(P<0.05)$. 


\section{CHF}

Incidence rates for CHF in patients with $\mathrm{PR}$ interval $\leq 200 \mathrm{~ms}$ or $>200 \mathrm{~ms}$ were 1.1 and 2.4 per 100 person-years respectively. Age, resting pulse rate, systolic blood pressure, physical inactivity, prior CAD, history of aspirin/ statin use, serum creatinine, hs-CRP, carotid IMT, QRS > $120 \mathrm{~ms}$, and PR prolongation $>200 \mathrm{~ms}$ were significant positive predictors of CHF (all $P<0.05$ ). Patients with PR prolongation $>200 \mathrm{~ms}$ had significantly reduced new-onset CHF-free survival (log rank: 5.0, $P=0.026$, Fig. 2d). Nevertheless, after adjustment for potential confounders, PR prolongation $>200 \mathrm{~ms}$ was no longer a significant predictor (HR: 1.29, 95\% CI: $0.54-3.11, P=0.56)$. Age, resting pulse rate, and history of aspirin use remained independent predictors of $\mathrm{CHF}$ (all $P<0.05$ ).

\section{Combined CV endpoints}

Incidence rates for combined $\mathrm{CV}$ endpoints in patients with PR interval $\leq 200 \mathrm{~ms}$ or $>200 \mathrm{~ms}$ were 2.6 and 6.3 per 100 person-years respectively. Age, prior CAD, smoking, systolic blood pressure, resting pulse rate, hsCRP, creatinine, history of ACEI/ARB use, QRS $>120 \mathrm{ms,}$ carotid IMT, and PR prolongation $>200 \mathrm{~ms}$ were all significant positive predictors for increased combined $\mathrm{CV}$ endpoints (all $P<0.05$, Additional file 1: Table S5). Kaplan-Meier analysis showed that patients with PR prolongation $>200 \mathrm{~ms}$ had significantly reduced combined endpoint-free survival (log rank: 14.2, $P<0.001$, Fig. 2e). Adjusted for potential confounders, PR prolongation $>200 \mathrm{~ms}$ remained an independent predictor for increased combined CV endpoints (HR: 1.95, 95\% CI: 1.11-3.43, $P=0.020$ ). The Fully-Adjusted Model yielded similar estimates (HR: 2.40, 95\% CI: $1.30-4.43, P=0.005$ ). Further adjustment for carotid IMT did not materially alter the independent prediction by PR prolongation (HR: 2.33, 95\% CI: $1.26-4.32, P=0.007)$. Other independent positive predictors included age, prior CAD, hs-CRP and creatinine (all $P<0.05$ ).

\section{Clinical utility in the prediction of CV events}

The clinical utility measures of sensitivity, specificity, positive predictive value (PPV) and negative predictive value (NPV) at specific cut-off points of PR interval in the prediction of each $\mathrm{CV}$ event were presented in Additional file 1: Table S6.

\section{Discussion}

This is our first recognized study to show that PR prolongation alone is a strong and independent predictor for CV death, new-onset ischemic stroke and MI, and combined $\mathrm{CV}$ endpoints including CHF among patients with $\mathrm{CAD}$ or risk equivalent. We also assessed the vascular phenotype of these high-risk $\mathrm{CV}$ patients and found that PR prolongation was independently related to increased carotid IMT. Interestingly, despite the stated observations, adjustment for carotid IMT did not materially alter the prediction of PR prolongation for incident $\mathrm{CV}$ events, which suggests that atherosclerosis alone does not fully explain the pathophysiological mechanism along the pathway of PR prolongation leading to clinical events.

The clinical implications of this study are multi-fold. Firstly, as opposed to the traditional school of thought that considered PR prolongation as a benign ECG feature [1], this study robustly showed that PR prolongation is strongly predictive of a collective plethora of pathophysiologically related $\mathrm{CV}$ outcomes, including $\mathrm{MI}$, ischemic stroke, $\mathrm{CHF}$ and $\mathrm{CV}$ death. Recent epidemiological studies (ARIC (2) and Framingham [3]) found higher risk of atrial fibrillation associated with PR prolongation. Our findings suggest that the pathophysiological implications of PR prolongation is far more than atrial fibrillation, and entails $\mathrm{MI}$, ischemic stroke and CHF, all of which could be commonly characterized by impaired systemic vascular function [7-9]. PR prolongation alone, regardless of other pertinent CV risk factors [12] such as those delineated in the CHADS2 scores [5], is a simple and independent CV risk predictor. Secondly, the magnitude of excess risk of PR prolongation was substantial for various adverse outcomes consistently (new-onset ischemic stroke: 4-fold excess risk; new-onset MI, 7-fold excess risk; CV death: 15-fold excess risk), thus reflecting PR prolongation has strong utility for $\mathrm{CV}$ prediction among high-risk patients. Thirdly, while the recent Heart and Soul Study [4] showed that PR prolongation predicted heart failure hospitalization and CV death amongst patients with stable CAD, our study further showed that PR prolongation has strong predictive applicability for $\mathrm{CV}$ events in a much wider spectrum of high-risk patients with prior CAD or other risk equivalents, including diabetes and stroke. Fourthly, the escalated risk of new-onset MI emerged at a lower PR interval cut-off of $>162 \mathrm{~ms}$, consistent with our prior exploratory study in terms of subclinical changes in endothelial dysfunction [6], and calls into question that early pathological changes may well precede the conventional cut-off of PR prolongation at $>200 \mathrm{~ms}$.

Mechanisms underlying our clinical observation are likely multi-dimensional. Firstly, PR prolongation representing delayed atrioventricular conduction could be an ECG manifestation of CV ageing [13]. Atherosclerosis is increasingly recognized as a multi-factorial degenerative disease that is closely related to the aging process. Our analysis indicated that PR prolongation could be an excellent clinical marker of CV ageing since adjustment for chronological age did not obliterate the prediction of carotid IMT and CV events by PR prolongation. Secondly, myocardial fibrosis may involve the cardiac conduction 
system and is closely linked to the presence of CV risk factors including CAD [14], hypertension [15], diabetes [16], hyperlipidemia [17] and inflammation [18]. Thus PR prolongation may be a risk marker that reflects the clustering of these important risk factors. Nevertheless, PR prolongation may also reflect higher underlying vagal tone [19], especially in the young [1], other than degenerative damage of the cardiac conduction system which predominantly affects the elderly [13]. In our study, comprehensive adjustment for important CV risk factors, even when including widened QRS complex signifying overt ventricular electrical dyssynchrony or widespread conduction system disease, did not abolish $\mathrm{PR}$ prolongation as an independent $\mathrm{CV}$ predictor. Therefore a third potential explanation arises: that PR prolongation may be causal to adverse CV events. Indeed, PR prolongation, be it vagal- or degenerative-predominant, may mediate its adverse vascular effects via increased intra-atrial pressure consequential to slowed atrioventricular conduction, resulting in neurohormonal activation [20]. Adverse neurohormonal changes including raised aldosterone may be reversible on sinus rhythm reversion [21]. Aldosterone is associated with a constellation of pro-atherosclerotic changes including raised inflammatory response [22], reduced circulating endothelial progenitor cell quantity and function [23] resulting in increased arterial stiffness [24]. Importantly, mineralocorticoid receptor blockade has been shown clinically to improve atherosclerotic changes [25]. Indeed, if neurohormonal activation is the explanatory mechanism that underlies PR prolongation-related vascular dysfunction, such findings may translate into potentially important preventive implications through pharmacological modulation of the neurohormonal system.

Vascular function as indicated by carotid IMT is a strong, independent and consistent predictor of both incident ischemic stroke and CV events overall among healthy subjects from diverse ethnicities [26]. Increased carotid IMT also predicts elevated risks of CV complications in patients with diabetes [27], hypertension [28] and prior atherosclerotic diseases [29]. In this study, the magnitude of increased carotid IMT by $+0.07 \mathrm{~mm}$ accounted for by PR prolongation is clinically significant, since each $0.1 \mathrm{~mm}$ increase of carotid IMT could translate into $18 \%$ excess risk of recurrent CV events [30]. On top of their greater susceptibility to $\mathrm{CV}$ events in general, patients with prior CAD also have increased risk of incident ischemic stroke, with diabetes being an additional independent predictor [31,32]. Our study further suggests that the adverse prognostic value of PR prolongation is independent and over that of carotid IMT for $\mathrm{CV}$ events in high-risk patients. Whether impaired vascular function is indeed along the pathophysiological pathway or simply a bystander will require further mechanistic studies. Future studies could be strengthened by incorporating multiple vascular assessment modalities to ensure consistent findings and scrutinise further mechanisitc insights.

It should be noted that from our study, the magnitude of risk associations of PR interval prolongation with new-onset $\mathrm{CV}$ events was striking, and that IMT did not fully explain such associations, suggesting that alternative mediating pathophysiological mechanisms could be present. Also, the HR estimates are relatively large. Despite our meticulous efforts in the study design and data analysis to carefully minimize biases or residual confounding, potential risk of bias and confounding cannot be excluded totally. Findings from the exploratory endpoint analyses should also best be verified in another independent study. Furthermore, the study of incident atrial fibrillation was not included in the current study, which should be explored in the future.

\section{Conclusions}

In this prospective study of patients with CAD or riskequivalent, PR prolongation strongly and independently predicts CV death, new-onset MI, ischemic stroke, and combined $\mathrm{CV}$ endpoints including CHF. Increased risk of MI emerged at a cut-off as low as PR interval $>162 \mathrm{~ms}$. Whether adverse vascular function in PR prolongation is an intermediate phenotype or represent a mediating pathway prior to clinical events warrants further investigations.

\section{Additional file}

\begin{abstract}
Additional file 1: Table S1. Univariable and Multivariable Predictors for Carotid IMT. Table S2. Univariable and Multivariable Predictors for Cardiovascular Death. Table S3. Univariable and Multivariable Predictors for New-Onset Ischemic Stroke. Table S4. Univariable and Multivariable Predictors for New-Onset Myocardial Infarction. Table S5. Univariable and Multivariable Predictors for Combined Cardiovascular Endpoints of New-Onset Myocardial Infarction, Ischemic Stroke, Congestive Heart Failure and Cardiovascular Death. Table S6. Estimates of Sensitivity (Se), Specificity (Sp), Positive Predictive Value (PPV) and Negative Predictive Value (NPV) of PR Interval in the Prediction for Cardiovascular Events. (DOC 444 kb)
\end{abstract}

\section{Abbreviations}

ACEl: Angiotensin-Converting Enzyme Inhibitors; BMl: Body-Mass Index; CAD: Coronary Artery Disease; CCB: Calcium Channel Blockers; CHF: Congestive Heart Failure; hbA1c: Glycosylated Hemoglobin A1c; HDL/LDL: High/Low-Density Lipoprotein; hs-CRP: High-Sensitivity C-Reactive Protein; IMT: Intima-Media Thickness; Ml: Myocardial Infarction

\section{Acknowledgements}

Not applicable.

\section{Funding}

The study was supported by CRCG Small Project Funding, University of Hong Kong (200907176063), and Sun Chieh Yeh Heart Foundation. Its study initiatives were part of the grant supported by the Hong Kong Research Grants Council Early Career Scheme (HKU 786012 M).

Availability of data and materials

Data and materials will not be shared to ensure strict confidentiality.

Authors' contributions

YHC originated the research hypothesis, was responsible for conceptual design and implementation of the research project, collected and analysed 
the data, and wrote the manuscript. LKK and KHY participated in data collection and study implementation; SWL participated in study design and supervised site management and patient recruitment; JJH, KHY, CPL and CWS supervised the study and critically refined the research paradigm; HFT supervised the overall research conceptualization and direction, study design and implementation, data collection and analysis, and writing of manuscript. All authors revised and approved the final submission.

\section{Ethics approval and consent to participate}

We declare that all patients completed written informed consent and the study was adherent to the Declaration of Helsinki and approved by the Ethics Committee, Hospital Authority (Hong Kong West)/ University of Hong Kong.

\section{Consent for publication}

We declare that all patients gave informed consent to the research participation and its publication.

\section{Competing interests}

The authors declare that they have no competing interests.

\section{Publisher's Note}

Springer Nature remains neutral with regard to jurisdictional claims in published maps and institutional affiliations.

\section{Author details}

'Division of Cardiology, Department of Medicine, Queen Mary Hospital, University of Hong Kong, Rm 1928, Block K, Hong Kong, China. ${ }^{2}$ Division of Neurology, University of Hong Kong, Hong Kong, China. ${ }^{3}$ Department of Medicine, Tung Wah Hospital, Hong Kong, SAR, China. ${ }^{4}$ Research Center of Heart, Brain, Hormone and Healthy Ageing, University of Hong Kong, Hong Kong, China.

\section{Received: 27 April 2017 Accepted: 17 August 2017}

Published online: 24 August 2017

\section{References}

1. Bexton RS, Camm AJ. First degree atrioventricular block. Eur Heart J. 1984; 5(Suppl A):107-9.

2. Wattanakit K, Folsom AR, Chambless LE, Nieto FJ. Risk factors for cardiovascular event recurrence in the Atherosclerosis Risk in Communities (ARIC) study. Am Heart J. 2005;149(4):606-12.

3. Cheng S, Keyes MJ, Larson MG, McCabe EL, Newton-Cheh C, Levy D, et al Long-term outcomes in individuals with prolonged PR interval or firstdegree atrioventricular block. JAMA. 2009;301(24):2571-7.

4. Crisel RK, Farzaneh-Far R, Na B, Whooley MA. First-degree atrioventricular block is associated with heart failure and death in persons with stable coronary artery disease: data from the Heart and Soul Study. Eur Heart J. 2011:32(15):1875-80.

5. Chan YH, Yiu KH, Lau KK, Yiu YF, Li SW, Lam TH, et al. The CHADS2 and CHA2DS2-VASc scores predict adverse vascular function, ischemic stroke and cardiovascular death in high-risk patients without atrial fibrillation: role of incorporating PR prolongation. Atherosclerosis. 2014;237(2):504-13.

6. Chan YH, Siu CW, Yiu KH, Li SW, Lau KK, Lam TH, et al. Abnormal vascular function in PR-interval prolongation. Clin Cardiol. 2011;34(10):628-32.

7. Kuvin JT, Patel AR, Sliney KA, Pandian NG, Rand WM, Udelson JE, et al. Peripheral vascular endothelial function testing as a noninvasive indicator of coronary artery disease. J Am Coll Cardiol. 2001;38(7):1843-9.

8. Kim J, Cha MJ, Lee DH, Lee HS, Nam CM, Nam HS, et al. The association between cerebral atherosclerosis and arterial stiffness in acute ischemic stroke. Atherosclerosis. 2011;219(2):887-91.

9. Borlaug BA, Olson TP, Lam CS, Flood KS, Lerman A, Johnson BD, et al. Global cardiovascular reserve dysfunction in heart failure with preserved ejection fraction. J Am Coll Cardiol. 2010;56(11):845-54

10. Chan YH, Lau KK, Yiu KH, Li SW, Chan HT, Tam S, et al. Isoflavone intake in persons at high risk of cardiovascular events: implications for vascular endothelial function and the carotid atherosclerotic burden. Am J Clin Nutr. 2007:86(4):938-45

11. Hara H, Oyenuga OA, Tanaka H, Adelstein EC, Onishi T, DM MN, et al. The relationship of QRS morphology and mechanical dyssynchrony to longterm outcome following cardiac resynchronization therapy. Eur Heart J. 2012:33:2680-91.
12. Barbero U, D'Ascenzo F, Nijhoff F, Moretti C, Biondi-Zoccai G, Mennuni M, et al. Assessing risk in patients with stable coronary disease: when should we intensify care and follow-up? Results from a meta-analysis of observational studies of the COURAGE and FAME era. Scientifica (Cairo). 2016;2016:3769152.

13. Fleg JL, Das DN, Wright J, Lakatta EG. Age-associated changes in the components of atrioventricular conduction in apparently healthy volunteers. J Gerontol. 1990;45(3):M95-100.

14. Oba T, Yasukawa H, Hoshijima M, Sasaki K, Futamata N, Fukui D, et al. Cardiacspecific deletion of SOCS-3 prevents development of left ventricular remodeling after acute myocardial infarction. J Am Coll Cardiol. 2012;59(9):838-52.

15. Kahan T. The importance of myocardial fibrosis in hypertensive heart disease. J Hypertens. 2012;30(4):685-7.

16. Asbun J, Villarreal FJ. The pathogenesis of myocardial fibrosis in the setting of diabetic cardiomyopathy. J Am Coll Cardiol. 2006;47(4):693-700.

17. Wendt N, Schulz A, Qadri F, Bolbrinker J, Kossmehl P, Winkler K, et al. Genetic analysis of salt-sensitive hypertension in Dahl rats reveals a link between cardiac fibrosis and high cholesterol. Cardiovasc Res. 2009:81(3):618-26.

18. Yang M, Zheng J, Miao Y, Wang Y, Cui W, Guo J, et al. Serum-glucocorticoid regulated kinase 1 regulates alternatively activated macrophage polarization contributing to angiotensin II-induced inflammation and cardiac fibrosis. Arterioscler Thromb Vasc Biol. 2012;32(7):1675-86.

19. Anselmino M, Ferraris F, Cerrato N, Barbero U, Scaglione M, Gaita F. Left persistent superior vena cava and paroxysmal atrial fibrillation: the role of selective radio-frequency transcatheter ablation. J Cardiovasc Med (Hagerstown). 2014;15(8):647-52.

20. Dixen U, Ravn L, Soeby-Rasmussen C, Paulsen AW, Parner J, Frandsen E, et al. Raised plasma aldosterone and natriuretic peptides in atrial fibrillation. Cardiology. 2007;108(1):35-9.

21. Wozakowska-Kaplon B, Bartkowiak R, Janiszewska G. A decrease in serum aldosterone level is associated with maintenance of sinus rhythm after successful cardioversion of atrial fibrillation. Pacing Clin Electrophysiol. 2010; 33(5):561-5.

22. Cooper JN, Tepper P, Barinas-Mitchell E, Woodard GA, Sutton-Tyrrell K. Serum aldosterone is associated with inflammation and aortic stiffness in normotensive overweight and obese young adults. Clin Exp Hypertens. 2012:34(1):63-70.

23. Thum T, Schmitter K, Fleissner F, Wiebking V, Dietrich B, Widder JD, et al. Impairment of endothelial progenitor cell function and vascularization capacity by aldosterone in mice and humans. Eur Heart J. 2011;32(10):1275-86.

24. Wu VC, Lo SC, Chen YL, Huang PH, Tsai CT, Liang CJ, et al. Endothelial progenitor cells in primary aldosteronism: a biomarker of severity for aldosterone vasculopathy and prognosis. J Clin Endocrinol Metab. 2011;96(10):3175-83.

25. Edwards NC, Steeds RP, Stewart PM, Ferro CJ, Townend JN. Effect of spironolactone on left ventricular mass and aortic stiffness in early-stage chronic kidney disease: a randomized controlled trial. J Am Coll Cardiol. 2009;54(6):505-12.

26. Rosvall M, Janzon L, Berglund G, Engstrom G, Hedblad B. Incidence of stroke is related to carotid IMT even in the absence of plaque. Atherosclerosis. 2005:179(2):325-31.

27. Wattanakit K, Folsom AR, Selvin E, Weatherley BD, Pankow JS, Brancati FL, et al. Risk factors for peripheral arterial disease incidence in persons with diabetes: the Atherosclerosis Risk in Communities (ARIC) study. Atherosclerosis. 2005:180(2):389-97.

28. Zanchetti A, Hennig M, Hollweck R, Bond G, Tang R, Cuspidi C, et al. Baseline values but not treatment-induced changes in carotid intima-media thickness predict incident cardiovascular events in treated hypertensive patients: findings in the European Lacidipine Study on Atherosclerosis (ELSA). Circulation. 2009;120(12):1084-90.

29. o Hartaigh B, Jiang CQ, Bosch JA, Zhang WS, Cheng KK, Lam TH, et al. Independent and combined associations of abdominal obesity and seated resting heart rate with type 2 diabetes among older Chinese: the Guangzhou Biobank Cohort Study. Diabetes Metab Res Rev. 2011;27(3):298-306.

30. Tsivgoulis G, Vemmos K, Papamichael C, Spengos K, Manios E, Stamatelopoulos K, et al. Common carotid artery intima-media thickness and the risk of stroke recurrence. Stroke. 2006;37(7):1913-6.

31. Ohira T, Shahar E, Chambless LE, Rosamond WD, Mosley TH Jr, Folsom AR. Risk factors for ischemic stroke subtypes: the atherosclerosis risk in communities study. Stroke. 2006:37(10):2493-8.

32. D'Ascenzo F, Barbero U, Moretti C, Palmerini T, Della Riva D, Mariani A, et al. Percutaneous coronary intervention versus coronary artery bypass graft for 
stable angina: meta-regression of randomized trials. Contemp Clin Trials. 2014;38(1):51-8.

Submit your next manuscript to BioMed Central and we will help you at every step:

- We accept pre-submission inquiries

- Our selector tool helps you to find the most relevant journal

- We provide round the clock customer support

- Convenient online submission

- Thorough peer review

- Inclusion in PubMed and all major indexing services

- Maximum visibility for your research

Submit your manuscript at www.biomedcentral.com/submit 